\title{
Diaphragmatic Involvement Should Not Preclude Curative-Intent Surgical Resection for Hepatocellular Carcinoma
}

\author{
Aslam Ejaz, MD, MPH, and Timothy M. Pawlik, MD, MPH, PhD \\ Department of Surgery, Division of Surgical Oncology, The Ohio State University Wexner Medical Center and James \\ Cancer Hospital and Solove Research Institute, Columbus, $\mathrm{OH}$
}

Hepatocellular carcinoma (HCC) is an aggressive malignancy with an incidence that varies worldwide. In addition to underlying liver disease severity, treatment decisions for HCC often are dictated by tumor-related factors such as size, differentiation, and local invasion. We therefore read with interest the study by Orimo and colleagues ${ }^{1}$ regarding the impact of HCC diaphragmatic involvement on patients undergoing curative-intent surgical resection.

The authors retrospectively analyzed a large cohort of 874 patients from Hokkaido University Hospital. ${ }^{1}$ Propensity score-matching was used to control for confounders to compare outcomes between patients undergoing hepatectomy for HCC who underwent diaphragmatic resection (DR) (5.3\%) and those who did not. Perhaps not surprisingly, HCC with diaphragmatic involvement was associated with more advanced tumorrelated factors including higher alpha-fetoprotein (AFP) levels, larger tumor size, and higher incidence of portal and hepatic vein invasion. After control for these and other measurable confounders, DR was not found to be independently associated with short- or long-term outcomes after hepatectomy. In fact, patients who underwent DR had outcomes similar to those of individuals in the non-DR cohort.

The aforementioned data were consistent with a previous report by Lin et al., ${ }^{2}$ who reported on the safety of DR for HCC as well as with other studies on en bloc resection

(C) Society of Surgical Oncology 2020

First Received: 9 June 2020;

Published Online: 23 June 2020

A. Ejaz, MD, MPH

e-mail: aslam.ejaz@osumc.edu of different primary tumors combined with adjacent structures. ${ }^{3-6}$ For example, Kimchi et al. ${ }^{3}$ reported on the safety and efficacy of combined pancreaticoduodenectomy and extended right hemicolectomy for locally advanced pancreatic adenocarcinoma, sarcoma, and colon cancer. In a separate study, Hunter et al. ${ }^{4}$ similarly noted that patient survival after en bloc resection of colon cancer adherent to other organs was comparable with long-term outcomes after standard colectomy for nonadherent colorectal cancers. In addition, other studies have similarly noted comparable short- and long-term outcomes among patients undergoing cytoreduction with and without DR for gynecologic malignancies. ${ }^{5,6}$

Collectively, the data suggest that technical aspects of the case (i.e., need to resect adjacent structures such as the diaphragm) do not drive long-term outcomes. Rather, as expected, tumor-related rather than technical-related factors have an impact on prognosis. These data support an aggressive surgical approach to achieve complete tumor extirpation for well-selected patients who have locally advanced disease, including individuals with HCC and diaphragmatic involvement.

Several shortcomings need to be considered when the data in the study by Orimo et al. ${ }^{1}$ are interpreted. The authors used propensity score-matching to balance the cohorts to minimize selection bias, which is ubiquitous in retrospective studies used to examine treatment differences. Notably, multiple clinicopathologic differences existed between the DR and non-DR cohorts before propensity score-matching. Specifically, the patients who underwent DR were older and more likely to have ChildPugh B cirrhosis than the patients who underwent hepatectomy without DR. Some biostatisticians have argued, however, that propensity score-matching can paradoxically increase confounder imbalance, thereby leading to 
estimates of exposure effects with greater bias. Although propensity score-matching may mitigate selection bias by balancing the cohorts on known covariates, unlike random assignment of treatments, the propensity score does not balance unobserved covariates. ${ }^{7}$ In addition, the issue of selection bias cannot be addressed with a "pre-selected" surgical data set. Specifically, the number of patients with diaphragmatic involvement who were not offered an operation during the study period remains unknown. In effect, the data can be generalized only to patients with HCC and diaphragm involvement who were very well-selected by experts such as the surgical team of Orimo and colleagues. ${ }^{1}$ As such, the true subset of patients with HCC and diaphragmatic involvement who benefit the most from surgical resection remains ill defined because the authors had already excluded a subset of patients a priori from the data set.

Another interesting difference between the DR and nonDR cohorts was that more than one fourth of the patients who did not need DR underwent a non-anatomic parenchymal-sparing hepatectomy versus only $2 \%$ of the patients in the DR cohort. These data are particularly interesting given that formal anatomic resection has been proposed as oncologically superior in the treatment of HCC given the tendency of $\mathrm{HCC}$ to invade portal tributaries. ${ }^{8}$ Notably, the authors also did not report data on margin status or width of the surgical margin. Such data would have been illustrative because margin status and margin width have been associated with long-term prognostic outcomes, including recurrence. ${ }^{9}$ The omission of these data was particularly noteworthy given that intrahepatic recurrence was more common among the patients in the non-DR cohort. Furthermore, the authors should have reported data on lung-specific complications (e.g., empyema, effusion, pneumonia, reintubation) and specific location of lymph node recurrence (e.g., intraabdominal vs mediastinal).

Treatment of HCC, especially large HCC or tumors that invade adjacent structures, remains challenging and should be determined in a multi-disciplinary setting. Despite the limitations of the study by Omino and colleagues, ${ }^{1}$ we agree that local invasion of adjacent structures, including the diaphragm, should not be considered a contraindication to resection of $\mathrm{HCC}$ when necessary to achieve a negative surgical margin. The data do highlight, however, that these patients frequently have other adverse biologic factors and remind us that biology, not surgery, is king.

DISCLOSURES The authors declare that they have no conflict of interests.

\section{REFERENCES}

1. Orimo $\mathrm{T}$, Kamiyama $\mathrm{T}$, Wakayama $\mathrm{K}$, et al. Hepatectomy combined with diaphragmatic resection for hepatocellular carcinoma with diaphragmatic involvement: a propensity scorematched analysis. Ann Surg Oncol. 2020. https://doi.org/10.1245/ s10434-020-08754-6.

2. Lin MC, Wu CC, Chen JT, Lin CC, Liu TJ. Surgical results of hepatic resection for hepatocellular carcinoma with gross diaphragmatic invasion. Hepato-gastroenterology. 2005;52:1497-501.

3. Kimchi ET, Nikfarjam M, Gusani NJ, Avella DM, StaveleyO'Carroll KF. Combined pancreaticoduodenectomy and extended right hemicolectomy: outcomes and indications. $H P B$. 2009;11:559-64.

4. Hunter JA, Ryan JA Jr, Schultz P. En bloc resection of colon cancer adherent to other organs. Am J Surg. 1987;154:67-71.

5. Aletti GD, Dowdy SC, Podratz KC, Cliby WA. Surgical treatment of diaphragm disease correlates with improved survival in optimally debulked advanced stage ovarian cancer. Gynecol Oncol. 2006;100:283-7.

6. Cliby W, Dowdy S, Feitoza SS, Gostout BS, Podratz KC. Diaphragm resection for ovarian cancer: technique and short-term complications. Gynecol Oncol. 2004;94:655-60.

7. Brooks JM, Ohsfeldt RL. Squeezing the balloon: propensity scores and unmeasured covariate balance. Health Services Res. 2013;48:1487-507.

8. Nakashima Y, Nakashima O, Tanaka M, Okuda K, Nakashima M, Kojiro M. Portal vein invasion and intrahepatic micrometastasis in small hepatocellular carcinoma by gross type. Hepatol Res. 2003;26:142-47.

9. Shi M, Guo RP, Lin XJ, et al. Partial hepatectomy with wide versus narrow resection margin for solitary hepatocellular carcinoma: a prospective randomized trial. Ann Surg. 2007;245:36-43.

Publisher's Note Springer Nature remains neutral with regard to jurisdictional claims in published maps and institutional affiliations. 